\title{
REPRESENTACIÓN DEL TEA \\ EN LOS MEDIOS DE COMUNICACIÓN: ANÁLISIS DE UN PERIÓDICO GENERALISTA
}

\section{Representation of ASD in mass media: analysis of a generalist newspaper}

\author{
Irene LACRUZ PÉREZ \\ Universidad de Valencia. Facultad de Magisterio. \\ Departamento de Didáctica y Organización Escolar \\ ilape2@alumni.uv.es \\ Raúl TÁrraga Mínguez \\ Universidad de Valencia. Facultad de Magisterio. \\ Departamento de Didáctica y Organización Escolar
}

Gemma Pastor Cerezuela

Universidad de Valencia. Facultad de Psicología. Departamento de Psicología Básica

M. Inmaculada Fernández AndRÉS

Universidad de Valencia. Facultad de Psicología. Departamento de Psicología Evolutiva $y$ de la Educación

Pilar SANz Cervera

Universidad de Valencia. Facultad de Magisterio.

Departamento de Didáctica y Organización Escolar

\section{Recepción: 23 de noviembre de 2018}

Aceptación definitiva: 6 de noviembre de 2019

RESUMEN: Los medios de comunicación influyen notablemente en sus consumidores, por lo que no son meros informadores, sino que también son creadores de opinión. Este hecho es relevante para el ámbito de la inclusión, en tanto que las necesidades específicas de apoyo educativo son también un tema tratado por los medios de comunicación. En el presente estudio se analizan los artículos que abordan la temática relacionada 
con el Trastorno del Espectro Autista (TEA) en el diario El País durante el año 2017. Los resultados del análisis de contenido de los artículos indican que el TEA fue un tema de interés en el año 2017. Los contenidos más recurrentes fueron las causas del TEA, frecuentemente desmintiendo su relación con la administración de vacunas, así como la visibilización del trastorno. En este sentido destaca que los aspectos que se dan a conocer del TEA son en su mayoría negativos. Asimismo, una de las carencias más significativas que se ha detectado respecto a la imagen que se proyecta del TEA es el hecho de que no se menciona de manera específica su heterogeneidad, una característica esencial del diagnóstico. En las conclusiones se discuten algunas implicaciones de los resultados para el ámbito de la inclusión.

Palabras Clave: medios de comunicación; noticias; proyección social; trastorno del espectro autista.

Aвsтract: Mass media influence significantly their users, so they don't just inform them but they generate opinions too. This fact is relevant to the field of inclusion, while the special educational needs are also an issue addressed by the media. In this study the articles that deal with the topic related to autism spectrum disorder (ASD) in the newspaper El Pais during the year 2017 are analysed. The results of the content analysis of the articles point out that ASD was a topic of interest during 2017. The most recurring contents were the causes of ASD, often denying their relation with the administration of vaccines, as well as the visibility of the disorder. In this sense, the study points out that the aspects that are shown about ASD are mostly negative. Additionally, regarding the projected image of ASD, one of the most significant deficiencies that has been figured out is the fact that its heterogeneity is not specifically mentioned, and it's an essential feature of the diagnosis. The conclusions discuss some implications of the results for the scope of inclusion.

KEY WORDs: autism spectrum disorder; mass media; news; social projection.

\section{Introducción}

$\tau$

OS MEDIOS DE COMUNICACIÓN DE MASAS constituyen un poderoso elemento en la difusión y creación de opinión en nuestra sociedad (Hanson, 2016). La selección de contenidos abordados en los medios, el tratamiento que se da a la información y las voces y mensajes que se amplifican o que se atenúan constituyen un elemento a partir del cual los consumidores de medios vamos conformando nuestra propia visión del mundo: nuestras actitudes, creencias, opiniones, ideologías, etc. (McCombs, 2018).

El ámbito de las necesidades específicas de apoyo educativo (NEAE) no escapa a esta influencia. La concepción social y las ideas que se asientan en el imaginario colectivo sobre un diagnóstico concreto van configurándose a través de un cóctel de fuentes de información compuesto por el posible conocimiento de casos puntuales; conversaciones informales; lecturas rigurosas más o menos esporádicas, y, por supuesto, los medios de comunicación, un elemento que, incluso de una manera poco 
consciente para nosotros, determina una parte significativa de nuestra manera de entender y percibir la realidad que nos rodea (Haller, 2010; Halliwell, 2017; Jones, 2014; Renwick, 2016). Así, la concepción social de las NEAE se construye sobre una amalgama de fuentes entre las que se pueden encontrar, en proporciones variables, tanto fuentes rigurosas y científicas, como fuentes carentes de ninguna validez científica o que ofrecen visiones parciales y sesgadas.

El Trastorno del Espectro Autista (TEA) es un diagnóstico especialmente expuesto a la influencia de los medios de comunicación por diferentes motivos. En primer lugar, los propios rasgos que caracterizan al TEA dificultan la inclusión social de las personas que lo presentan e incluso suscitan el interés de la sociedad sobre ellos, pues según lo establecido en el DSM-V (APA, 2013) el TEA es un trastorno del neurodesarrollo caracterizado principalmente por déficits persistentes en interacción y comunicación social, así como por patrones repetitivos y restringidos de conducta, actividades e intereses. Además, frecuentemente presentan dificultades en el desarrollo del lenguaje oral. En segundo lugar, se trata de un diagnóstico cuya prevalencia ha aumentado exponencialmente durante los últimos años (Christensen et al., 2016), lo cual ha provocado que se incremente su presencia de manera significativa en los medios de comunicación. Por último, es un diagnóstico que aparece de manera recurrente en productos de consumo cultural de masas, como películas, series de TV y libros que, debido probablemente a las características del propio diagnóstico, consideran el TEA como un asunto que genera fascinación o incluso que posee cierto carácter enigmático (Morgan, 2018; Murray, 2007).

Por tanto, es interesante analizar cómo es la imagen que los medios de comunicación proyectan a sus consumidores sobre el TEA, ya que a partir de esta proyección se conforman algunas cuestiones tan relevantes en el terreno de la inclusión como la difusión de una información rigurosa y carente de sesgos sobre el propio diagnóstico y los mecanismos de intervención (Belcher y Maich, 2014; Lanovaz, Dufour y Shah, 2015). En definitiva, son un elemento a partir del cual se conforman buena parte de las actitudes que los ciudadanos construyen sobre el TEA (Brewer, Zoanetti y Young, 2017; White, Meloy, Mohandie y Kienlen, 2017).

Al realizar una revisión de trabajos a nivel internacional en los que se analiza la imagen que ofrece la prensa sobre el autismo, podemos observar cómo los medios de comunicación construyen discursos que se posicionan a favor o en contra de la inclusión educativa de los niños con TEA. Por ejemplo, Dorries y Haller (2001) llevaron a cabo un análisis de la cobertura mediática que se produjo en Estados Unidos sobre la batalla legal que mantuvo la familia de un niño con TEA para conseguir su escolarización en un centro educativo ordinario. Durante 4 años de publicación de noticias en prensa escrita sobre el caso, se construyeron diferentes discursos en torno a los costes y beneficios de la inclusión que generaron un debate social de alcance nacional, en el que los medios se posicionaban "a favor" o "en contra” de los principios de la inclusión educativa, proporcionando argumentos que muchas veces chocaban frontalmente con los de la postura contraria, y que se convertían en un elemento potente para crear opiniones sobre este tema en los lectores.

En un estudio más amplio realizado en Australia, que consistió en una revisión de las publicaciones sobre autismo en prensa escrita entre 1996 y 2005, Jones y Harwood 
(2009) obtuvieron varias conclusiones interesantes. El análisis diacrónico de la presencia del TEA en la prensa escrita evidenció un aumento exponencial del número de artículos publicados cada año durante la década estudiada (desde 9 artículos en 1996 hasta más de 300 en 2005), lo que denota el interés creciente por parte de la prensa en el tema del autismo. Asimismo, la revisión de las noticias publicadas en prensa escrita mostró que, durante los años 2004 y 2005, el número de noticias que ofrecían una visión negativa del autismo fue sensiblemente superior al número de noticias que ofrecían una visión positiva. Entre las noticias negativas destacaban aspectos como el fuerte impacto familiar que suponía el diagnóstico, o se informaba de sucesos relacionados con situaciones de abandono de niños con TEA por parte de sus familias o de crímenes cometidos por personas con TEA.

Precisamente, el hecho de relacionar el TEA con sucesos violentos en los medios de comunicación ha sido ampliamente estudiado en EE. UU. (Brewer et al., 2017; White et al., 2017). Este análisis ha evidenciado que el supuesto vínculo entre autismo y violencia puede influir en la creación de actitudes de miedo o rechazo hacia las personas con TEA, cuando en realidad este binomio TEA-violencia carece de cualquier tipo de justificación científica rigurosa.

También en China se han realizado estudios que analizan la visión que se ofrece del TEA en la prensa escrita. Bie y Tang (2015) analizaron la cobertura que los medios de comunicación hicieron sobre el TEA entre 2003 y 2012. En esta revisión también se evidenció un aumento del número de noticias relacionadas con el autismo, de proporciones similares al caso de Australia, e igualmente se detectó que la visión que se ofrecía del TEA en la prensa era preferentemente de carácter negativo, haciendo un énfasis especial en la carga que supone para las familias la presencia de un niño con TEA.

Pero quizá el estudio que más claramente ha mostrado la gran capacidad de influencia de la prensa escrita en la conformación de actitudes hacia el TEA es el que llevaron a cabo Briant, Watson y Philo (2013). Los citados autores revisaron las noticias publicadas en prensa escrita en Reino Unido durante los años 2010 y 2011 (años fuertemente marcados por las decisiones políticas sobre ajustes presupuestarios del período más duro de la crisis económica) con las noticias aparecidas en los mismos medios de comunicación en los años 2004 y 2005 (período previo a la crisis económica). El análisis de Briant et al. (2013) evidencia un cambio en el enfoque que se ofrecía sobre la discapacidad en la prensa escrita coincidiendo con el inicio de la crisis económica. La cobertura mediática que se realizaba en los años 2010 y 2011 de la discapacidad tenía un carácter mucho menos empático con esta que en el período anterior a la crisis. Hubo un considerable aumento de noticias en las que se denunciaban casos de fraude relacionados con las ayudas públicas a las familias de personas con discapacidad, un aumento del lenguaje peyorativo hacia las personas con discapacidad, y se construyó una imagen de la discapacidad que los autores del estudio denominan folk devil, en referencia a la demonización pública que se ofrecía en la prensa de las personas con discapacidad.

En nuestro contexto, hasta la fecha no se ha llevado a cabo ninguna revisión sistemática sobre el tratamiento del TEA en la prensa, aunque sí se han realizado varios 
estudios que analizan de diferente manera el tratamiento de la discapacidad en medios escritos. Entre estos estudios, destaca el llevado a cabo por Cuesta, Marchena y Aguiar (2015), quienes analizaron el enfoque que se daba a la información relacionada con la discapacidad en los diarios $A B C$ y $L a$ Vanguardia durante varios meses de los años 1978, 1995 y 2007, con la intención de analizar los posibles cambios de enfoque en las tres fechas. En este estudio se clasificaron las noticias que aparecían en la prensa de acuerdo a tres modelos de discapacidad: el modelo tradicional, en el que se presenta la discapacidad como una carga para la familia, se subestiman las capacidades de las personas diagnosticadas o se relaciona la discapacidad con aspectos como la delincuencia y el vandalismo; el modelo rehabilitador, en el que la discapacidad se concibe como una etiqueta negativa, que requiere de una atención especializada por parte de profesionales de la salud, preferiblemente en contextos segregados y se critican las experiencias inclusivas; y el modelo social, en el que se destaca la posibilidad de llevar una vida independiente y normalizada, se enfatiza la necesidad de asegurar el cumplimiento de los derechos de las personas con discapacidad y se incluyen noticias en que se da muestra de ejemplos de superación personal.

Los resultados del estudio mostraron que, en los artículos revisados de los años 1978 y 1995, más de la mitad de las noticias publicadas por estos medios de comunicación relacionadas con la discapacidad cumplían los cánones del modelo tradicional. Aunque afortunadamente también se evidenció que, en los dos medios de comunicación revisados, las noticias que se adaptaban al enfoque del modelo social fueron ascendiendo en los tres momentos analizados, aunque todavía no se ha conseguido revertir totalmente el enfoque negativo que se daba a la discapacidad.

En otro estudio llevado a cabo en nuestro contexto (González, Montes y Paniagua, 2018), se solicitó a través de entrevistas una valoración a un grupo de periodistas (varios de ellos con discapacidad) sobre la presencia y el tratamiento que las diferentes televisiones otorgan a la discapacidad en nuestro país. Los resultados del estudio mostraron una diferencia significativa entre las televisiones públicas, en las que existen programas específicos que abordan diferentes aspectos relacionados con la discapacidad, y las televisiones privadas, en las que no existe ningún espacio similar. Sin embargo, la valoración que hicieron los propios periodistas sobre estos espacios televisivos distaba mucho de ser positiva, ya que reseñaron diferentes carencias e incluso los calificaron como una manera de "maquillar" una evidente despreocupación por la discapacidad en las televisiones públicas.

Gómez, Martín y Castellet (2014) realizaron otro estudio en nuestro país en el que revisaron 6 libros de estilo de algunos de los principales medios de comunicación nacionales para analizar las cuestiones relativas a la discapacidad que aparecían en dichos manuales de estilo. Los resultados de este estudio mostraban la presencia de algunas recomendaciones interesantes, aunque desgraciadamente su cumplimiento no es ni mucho menos generalizado en los medios de comunicación. Entre estas recomendaciones se encuentran: tratar de eliminar en el lenguaje las connotaciones negativas asociadas a la discapacidad, evitar definir a las personas por su discapacidad $\mathrm{o}$, incluso, mencionar la presencia de la discapacidad únicamente cuando esta es un hecho relevante en la información que se está proporcionando. 
Finalmente, la Asociación Española de Profesionales del Autismo (AETAPI, 2011) publicó un protocolo en el que se recogen diferentes sugerencias a los medios de comunicación para que traten el TEA ofreciendo a sus lectores o espectadores una visión objetiva, positiva y no sensacionalista del autismo. En este sentido, apuntan que los profesionales que elaboran las noticias referentes al TEA, o a cualquier tipo de discapacidad, deberían estar sensibilizados, formados e incluso especializados en esta materia para así evitar, entre otras cosas, la transmisión de falsas creencias. Por ello en este mismo documento se recogen algunos de los mitos que existen sobre el TEA y que los medios de comunicación en general han contribuido a perpetuar. Un ejemplo de esto es el relativo a las causas del trastorno, pues su aparición no puede reducirse a un único factor y mucho menos a la falta de cariño parental. Otra creencia errónea que se desmiente es la relativa a la capacidad intelectual de las personas con TEA, pues se tiende a pensar que esta es superior a la media de la población (tomando de referencia el Síndrome de Asperger), cuando es frecuente que las personas con TEA presenten discapacidad intelectual u otro trastorno asociado.

Dada la escasez de trabajos a nivel nacional dedicados a analizar la imagen que se ofrece desde los medios de comunicación sobre la discapacidad y dado que, hasta donde conocemos, ninguno de estos trabajos ha analizado de manera específica el TEA, los objetivos que se plantean en este trabajo son analizar en términos cuantitativos la presencia del TEA en un periódico generalista nacional durante el año 2017 y analizar la imagen que este medio de comunicación proyecta acerca del TEA, así como sus posibles repercusiones en el ámbito de la educación inclusiva.

Consideramos que estos objetivos pueden ser interesantes por, al menos, dos motivos fundamentales. En primer lugar, se trata del primer estudio en nuestro país que aborda de una manera sistemática el análisis del tratamiento que la prensa escrita nacional da al TEA, un tema que es necesario analizar debido al aumento de la presencia de este diagnóstico en los medios de comunicación. Por otro lado, creemos que los resultados del estudio pueden proporcionar algunas de las claves sobre la manera en que los medios de comunicación influyen en la generación de opiniones y actitudes sobre el TEA, un ámbito de gran relevancia para alcanzar mejoras en la inclusión social de las personas con TEA.

\section{Metodología}

\subsection{Selección de la muestra}

En el presente estudio se analizan los artículos referentes al TEA publicados en el diario El País (edición digital), durante el año 2017. Se ha escogido este periódico debido a su posición como líder de la prensa digital en España.

Las palabras clave introducidas para la búsqueda de artículos fueron autismo y trastorno del espectro autista. La búsqueda se limitó a los artículos publicados durante el año 2017.

Tras las búsquedas, se descartaron los artículos repetidos o publicados en diferentes idiomas y los artículos en los que el TEA no era el elemento central del texto. 
La Figura 1 muestra el proceso realizado de búsqueda y selección de artículos hasta reducir a los 77 artículos que conforman la presente revisión.

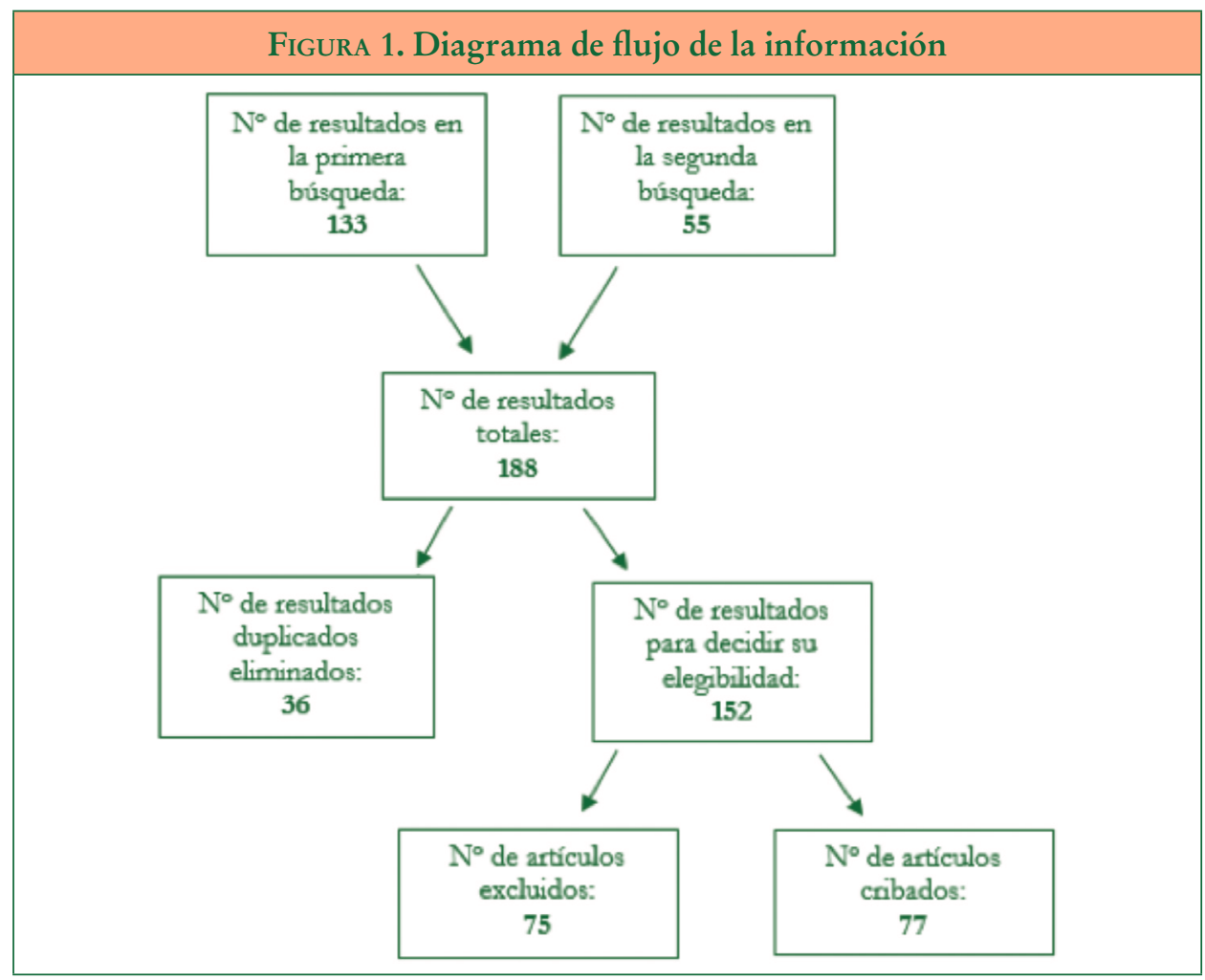

\subsection{Análisis de contenido}

El establecimiento de las categorías para realizar el análisis de contenido se realizó siguiendo el procedimiento de codificación emergente, de acuerdo a las recomendaciones de Stemler (2001). Los dos primeros autores del estudio llevaron a cabo, de manera independiente, un análisis preliminar de los artículos seleccionados. A partir de este análisis inicial cada autor elaboró un listado de indicadores de los artículos para conformar las categorías. Tras este proceso de confrontación de listados se alcanzó un consenso para clasificar los artículos en función de dos variables fundamentales: tipo de contenido abordado e imagen social proyectada.

En cuanto al contenido abordado en la noticia, se detectaron cuatro temas principales: 1) causas del TEA; 2) descripción conductual del TEA; 3 ) visibilización (acciones llevadas a cabo para dar a conocer la existencia del TEA y su problemática asociada); y 4) intervención. 
En cuanto a la imagen social que se proyecta del TEA, a partir del contenido de los artículos, se han establecido seis categorías diferentes: 1) vulnerable, en el caso de que se presenten como personas con riesgo a la exclusión social; 2) genio, cuando se habla solo de aquellos con capacidades intelectuales superiores a la media; 3) precisa atención especial, cuando se habla de las personas con autismo como aquellas que necesitan un trato particular; 4) dificultades sociales, cuando se muestran principalmente como individuos que no establecen ninguna interacción social y tienen dificultades para la comprensión de emociones; 5) ejemplo de superación, cuando se narran historias de vida de personas a quienes el trastorno se les presentaba como una gran barrera y progresivamente han obtenido grandes resultados; y 6) impersonal, incluye todos los artículos que se centran en el origen del TEA sin hablar de las personas que lo presentan, es decir, aquellos planteados desde un enfoque puramente científico.

Una vez acordadas estas categorías, los dos primeros autores del estudio llevaron a cabo una codificación independiente de los artículos incluidos en la presente revisión. A partir de esta codificación independiente se calculó el índice kappa de Cohen para las dos variables en que se categorizaron los artículos (tipo de contenido e imagen social proyectada). El resultado para la variable tipo de contenido fue $x=1.00$ y para la variable imagen social proyectada $x=.91$ Ambos valores indican un nivel de concordancia casi perfecto, de acuerdo a la interpretación del estadístico propuesta por Landis y Koch (1977). En los casos en que no hubo coincidencia entre los análisis realizados de manera independiente por los dos autores, la codificación final de los artículos se realizó tras alcanzar una decisión consensuada entre ambos.

\section{Resultados}

El análisis de la primera variable, el tema central de cada noticia, dio lugar a los resultados reflejados en la Tabla 1.

El contenido más frecuente en los artículos analizados hace referencia a las causas del TEA (31 de los 77 artículos). De ellos, 26 artículos se centran específicamente en desmentir una falsa creencia bastante arraigada, que sostiene que el TEA está relacionado con la administración de vacunas durante la primera infancia. En el resto de artículos de esta categoría, el origen del TEA se plantea como desconocido, aunque se le otorga un peso importante a la genética y, en menor medida, a factores ambientales.

\begin{tabular}{|l|c|}
\hline \multicolumn{2}{|c|}{ TABLA 1. Tipo de contenido abordado } \\
\hline \multicolumn{1}{|c|}{ TEMA } & N. $^{\circ}$ ARTÍculOs (\%) \\
\hline Causas & $31(40 \%)$ \\
\hline Visibilización & $25(33 \%)$ \\
\hline Intervención & $17(22 \%)$ \\
\hline Descripción conductual & $4(5 \%)$ \\
\hline
\end{tabular}

La categoría de visibilización incluye artículos que principalmente tienen dos objetivos: 1) dar a conocer las características del TEA y 2) mostrar las diferentes 
problemáticas a las que deben hacer frente las personas con TEA y sus familias. Así, este grupo de artículos comprende noticias sobre series televisivas en las que uno de los protagonistas principales presenta rasgos de TEA (esto ocurre en 6 de las 25 noticias de este bloque), reportajes dedicados en exclusiva a dar a conocer el TEA o bien noticias sobre experiencias que tienen lugar en diferentes países o ciudades de España como sesiones de cine adaptadas a las necesidades de niños con TEA.

Los artículos incluidos en la categoría de intervención tratan los beneficios que supone un diagnóstico precoz del trastorno; propuestas de tratamiento como la equinoterapia, la musicoterapia, el yoga o el judo, así como pautas a seguir para llevar a cabo una inclusión educativa efectiva.

Finalmente, hay un reducido número de artículos en los que el tema central relacionado con el TEA es la realización de una descripción conductual del diagnóstico.

En cuanto a la imagen social proyectada del TEA, el análisis de contenido ofrece los resultados que se recogen en la Tabla 2.

Esta categoría muestra que aproximadamente el $36 \%$ de los artículos analizados ofrecen una visión impersonal del TEA (nuevamente debido a que hay una gran cantidad de artículos que tratan el tema de la vacunación), mientras que el $64 \%$ restante aborda el TEA desde un punto de vista más personalizado, proyectando una imagen del diagnóstico que destaca la vulnerabilidad social, genialidad, necesidad de atenciones especiales, rasgos antisociales o ejemplo de superación que presentan algunas personas con TEA.

\begin{tabular}{|c|c|}
\hline ASPECTO DESTACADO & N. ${ }^{\circ}$ ARTÍ́culOS (\%) \\
\hline Impersonal & $28(36 \%)$ \\
\hline Vulnerable & $13(17 \%)$ \\
\hline Genio & $12(15 \%)$ \\
\hline Precisa atención especial & $10(13 \%)$ \\
\hline Dificultades sociales & $9(12 \%)$ \\
\hline Ejemplo de superación & $5(7 \%)$ \\
\hline
\end{tabular}

\section{Discusión}

Los objetivos del presente estudio giraban en torno a realizar un análisis cuantitativo del número de noticias relacionadas con el TEA aparecidas durante un año en un periódico generalista nacional, y analizar la imagen que este medio de comunicación proyecta acerca del TEA. Una vez obtenidos los resultados, consideramos que, a partir del análisis de estos resultados, podemos extraer al menos cuatro conclusiones relevantes.

En primer lugar, si analizamos el número de artículos publicados sobre esta temática, podemos llegar a la conclusión de que el TEA es un tema de actualidad. En 2017 se publicaron un total de 77 artículos en los que se trataba alguna circunstancia 
relacionada con el TEA como uno de los constructos centrales del artículo. Ello supone aproximadamente una noticia cada 5 días durante todo un año, una cantidad de información relevante que contribuye a que los consumidores de medios de comunicación tengan un cierto conocimiento sobre el TEA. Esta información proveniente de los medios de comunicación es uno de los elementos a partir del cual los consumidores conformamos nuestra concepción de la realidad social y también nuestras actitudes hacia esta realidad (McCombs, 2018). Dado que la inmensa mayoría de las noticias analizadas tiene una extensión breve (se trata de noticias o crónicas que se leen en unos pocos minutos), es necesario analizar qué aspectos del TEA se destacan en estos textos breves y cuáles se silencian implícitamente, ya que a través de este análisis tendremos un indicador que puede ayudar a explicar buena parte del modo en que el TEA es percibido socialmente.

Precisamente la segunda conclusión gira en torno a este análisis. La clasificación en temáticas obtenida en los resultados muestra que la temática que más se ha repetido durante el último año es la relacionada con desmentir el vínculo entre vacunación y TEA. Hasta la fecha, todos los estudios rigurosos que se han llevado a cabo para tratar de buscar esta relación han sido infructuosos. Pese a ello, desgraciadamente, la (falsa) creencia de que el TEA está causado por la administración de vacunas a bebés y niños es una idea relativamente arraigada en una parte de la población, e incluso en profesionales de la educación o futuros profesionales de la educación (Hebert y Koulouglioti, 2010). Quienes defienden esta relación entre autismo y vacunas lo hacen apoyándose en una hipotética correlación temporal entre el inicio de la sintomatología autista y el período en que habitualmente se administran determinadas vacunas de acuerdo al calendario de vacunación de la mayoría de sistemas sanitarios nacionales. Sin embargo, se trata únicamente de una correlación en el tiempo en algunos casos de niños con TEA, de la cual no podemos asumir que se derive causalidad. Desde hace décadas, numerosos estudios han analizado de manera específica esta relación entre vacunas y autismo y en todos los casos han descartado que exista relación de causalidad entre ambos, obteniéndose resultados con indicadores estadísticos robustos (Doja y Roberts, 2006; Taylor, Swerdfeger y Eslick, 2014; Wilson, Mills, Ross, McGowan y Jadad, 2003).

Lamentablemente, no se trata de un simple error conceptual limitado únicamente al plano teórico. La creación de dudas, confusión o incluso la divulgación de información errónea sobre este asunto pueden llegar a suponer también un riesgo para la salud pública, ya que las vacunas que están bajo sospecha de estar relacionadas con el autismo han sido tremendamente eficaces para reducir los índices de mortalidad infantil en los países en que se administran mediante campañas de vacunación universales (Kumanan et al., 2003). Noticias como las publicadas el año 2017 en el diario El País, que hasta en 26 ocasiones en un año (es decir, aproximadamente una vez cada dos semanas) ofreció información que desmentía esta relación, parece que deberían ayudar a desmitificar esta falsa creencia. Sin embargo, también es posible que la abundancia de noticias para desmentir una mentira genere, paradójicamente, un efecto de ruido, que contribuye a que se continúe hablando de esta falsa creencia, dándole así posibilidades de permanecer de actualidad. 
En cuanto a las otras temáticas tratadas, destacan las noticias que tienen como uno de sus objetivos el dar a conocer el TEA a los lectores, bien a través de artículos en que se exponen las dificultades que deben afrontar las personas con TEA y sus familias, o bien a través de artículos en que se destaca la presencia del TEA en otros productos culturales, como las series de TV y películas. De esta manera, el propio diario de información se hace eco de que el TEA se está convirtiendo en un elemento que, tal y como exponen numerosos trabajos científicos, despierta interés e incluso fascinación entre la población (Morgan, 2018; Murray, 2007). Este elevado interés parece que es aprovechado por los creadores de productos culturales para introducir el TEA como un elemento relevante en películas y series de TV.

En tercer lugar, en cuanto a la imagen que los artículos analizados proyectan del TEA, encontramos que, en un porcentaje relevante de ocasiones (un $36 \%$ ), la temática del TEA se aborda de una manera impersonal en la que se trata únicamente información sobre el diagnóstico (como se ha indicado anteriormente, en la mayoría de los casos se trata de artículos relacionados con las vacunas) y no aparece ningún protagonista de la noticia en primera persona.

En el resto de artículos se ofrece una proyección del TEA dispar. Aproximadamente un $65 \%$ de los artículos restantes resaltan aspectos negativos sobre el TEA, como su vulnerabilidad, la necesidad de atención o las dificultades en las relaciones sociales. Mientras que aproximadamente un $35 \%$ destacan aspectos positivos, como la genialidad de algunas personas con TEA en un ámbito concreto o el destacarse como ejemplos de superación.

$\mathrm{El}$ análisis de estos datos nos lleva a pensar que, aunque el tratamiento que se da al TEA contempla también una imagen positiva, son más numerosos los artículos en que se destacan los aspectos negativos. Este resultado es semejante al que se ha obtenido en otros estudios similares a este realizados a nivel internacional (Bie y Tang, 2015; Jones y Harwood, 2009), en los que se han destacado aspectos negativos del TEA, como la carga familiar que supone el diagnóstico de este trastorno; las dificultades que encuentran las personas con TEA para participar en la sociedad mediante el trabajo o el ocio, o incluso la asociación de TEA y violencia (Brewer et al., 2017; White et al., 2017).

Pese a ello, los resultados también muestran la presencia de noticias en las que se destacan aspectos positivos del TEA, como en los artículos que presentan a personas con TEA a quienes dibujan como ejemplos de superación. Estas noticias entrarían dentro de la categoría de modelo social, de acuerdo a la clasificación que realizaron Cuesta et al. (2015) en su análisis de la prensa española, y confirman que, tal y como se vio en ese estudio, el TEA también es tratado de manera positiva en la prensa escrita.

Finalmente, consideramos que, además de analizar los aspectos que se destacan en la prensa sobre el TEA, también es necesario analizar qué aspectos relevantes del TEA no se han tratado en los artículos analizados. A este respecto, probablemente quizá el aspecto que más llame la atención es que prácticamente en ninguno de los artículos revisados se tiene en cuenta una de las características fundamentales del TEA: la heterogeneidad. Una de las palabras del propio nombre del diagnóstico, espectro, nos da una idea de que existe un amplísimo abanico de características en las personas con TEA, 
unas características que se agrupan en torno a las dificultades en la comunicación e interacción social y a la presencia de comportamientos e intereses restringidos, repetitivos y estereotipados, pero que pueden presentar manifestaciones muy distintas en diferentes personas e incluso en una misma persona a lo largo del tiempo.

En muchos artículos se personaliza en casos concretos, destacándose de ellos una característica particular (un problema médico asociado, dificultades en la adaptación escolar, un talento específico...). Pero existen pocos casos de artículos en que se explique que el TEA es un diagnóstico tremendamente heterogéneo, que puede darse en casos con niveles de severidad muy dispares. Por ejemplo, un niño pequeño que no es capaz de comunicarse con palabras, que rechaza cualquier contacto con adultos diferentes de su entorno más inmediato y que presenta rabietas frecuentes cuya causa es difícil de conocer presenta TEA; pero también presenta TEA un joven que estudia en la universidad y es brillante en su ámbito de estudio, aunque requiere de apoyos para relacionarse con compañeros, entender situaciones sociales cotidianas o utilizar elementos de la comunicación no verbal en su día a día. Ambos casos comparten el diagnóstico TEA, pero obviamente ambos son muy diferentes entre sí.

En los artículos analizados, encontramos casos concretos de personas con TEA que ayudan a los lectores a hacerse una idea del día a día de las personas con este tipo de diagnóstico. Sin embargo, son pocos los artículos en los que expresamente se menciona la heterogeneidad del TEA, por lo que es posible que los lectores se formen la idea de que los casos acerca de los que han leído representan todo el espectro, cuando en realidad no es así.

\section{Limitaciones del estudio, futuras líneas de investigación e implicaciones prácticas}

El presente trabajo tiene algunas limitaciones que invitan a continuar trabajando en el futuro: en primer lugar, tan solo se ha analizado un medio de comunicación, por lo que no se trata de un estudio representativo de todos los medios de comunicación, ni siquiera de toda la prensa. Por tanto, es necesario continuar trabajando para completar este análisis con otros medios de comunicación (escritos y audiovisuales), para ofrecer una visión más completa de la imagen que los medios están transmitiendo sobre el TEA.

Además, tal y como se ha comentado anteriormente, estudios previos como el de Murray (2007) o el de Morgan (2018) constatan que el TEA está presente en numerosas series y películas que se producen en la actualidad, lo cual abre la puerta a futuras investigaciones en las que se analice la visión que se da del TEA también en estos productos culturales. El mundo del cine y las series de TV, los canales de vídeos en streaming, o incluso los blogs y páginas web, son elementos que influyen también de una manera decisiva en las concepciones sobre el TEA, por lo que sería interesante realizar un análisis de estos medios en futuros trabajos.

Finalmente, el presente estudio tiene implicaciones claras en al menos tres ámbitos: la formación del profesorado, la práctica educativa diaria en escuelas e institutos y la inclusión social y moral de las personas con TEA. 
Los estudiantes que actualmente se encuentran inmersos en su formación inicial como maestros pertenecen a una generación que consume información y productos culturales a través de medios muy variados (medios de comunicación tradicionales, redes sociales, plataformas de vídeo, etc.). Tal y como hemos visto en este trabajo, el TEA está presente en la prensa, pero la imagen que se proporciona del mismo no es completa, sino parcial, y en muchas ocasiones se ofrece una visión negativa. Los formadores de profesores debemos conocer qué tipo de información sobre el TEA están recibiendo los futuros docentes a través de estos medios, para de esta manera planificar correctamente qué falsas informaciones debemos desmentir, qué aspectos silenciados del TEA debemos destacar y fundamentalmente qué claves debemos proporcionar para consumir de manera crítica los medios de comunicación y otros productos culturales.

Respecto a la práctica educativa diaria en escuelas e institutos, si queremos verdaderamente alcanzar niveles elevados de inclusión debemos estar atentos a qué tratamiento se da al TEA (y en realidad también al resto de alumnos y alumnas con NEAE) en los medios de comunicación, ya que de esta manera estaremos en condiciones de desmentir las informaciones claramente incorrectas que se ofrecen en ocasiones, matizar o completar informaciones que, siendo correctas, solo ofrecen una visión parcial e incompleta del TEA, y podremos tratar de educar a nuestros estudiantes también en un consumo crítico de medios de comunicación. De esta manera, formando maestros críticos, que a su vez forman estudiantes críticos con los medios de comunicación, consideramos que estaremos en mejor disposición para crear una sociedad más justa e inclusiva para todos.

Finalmente, garantizar no solo la inclusión educativa, sino también la inclusión social y moral de las personas con TEA, y en general de todas las personas con cualquier tipo de diversidad funcional, exige una transformación sistémica. Esto supone cambiar radicalmente el enfoque y la organización del actual sistema social para enfocarlo desde el derecho inalienable que tienen todas las personas a una vida digna (Urien, 2016). Los medios de comunicación juegan un papel crucial en dicha transformación por su poder de construir una representación social del TEA. De esta imagen proyectada derivan una serie de actitudes hacia las personas con TEA que pueden afectar gravemente a su autoconcepción, pues de las conductas de los demás hacia uno mismo depende en gran medida la percepción de que la vida propia es igual de valiosa que la de cualquier otro (Urien, 2016).

\section{Referencias bibliográficas}

American Psychiatric Association (2013). Diagnostic and statistical manual of mental disorders ( $5^{\text {th }}$ ed.). Arlington, VA: American Psychiatric Publishing.

Asociación Española de Profesionales del Autismo (2011). Recomendaciones profesionales a los medios en el tratamiento del autismo. Consultado en febrero de 2019 en http:// aetapi.org/download/protocolo-comunicacion-aetapi-2/?wpdmdl=3311.

Belcher, C. y Maich, K. (2014). Autism spectrum disorder in popular media: storied reflections of societal views. Brock Education: A Journal of Educational Research and Practice, 23(2), 97-115. https://doi.org/10.26522/BROCKED.V23I2.311. 
REPRESENTACIÓN DEL TEA EN LOS MEDIOS DE COMUNICACIÓN: ANÁLISIS DE UN PERIÓDICO GENERALISTA IRENE LACRUZ PÉREZ, RAÚL TÁRRAGA MÍNGUEZ, GEMMA PASTOR CEREZUELA,

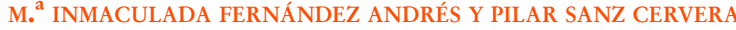

BIE, B. y TANG, L. (2015). Representation of autism in leading newspapers in China: A content analysis. Health communication, 30(9), 884-893. https://doi.org/10.1080/10410236.2014.8 89063.

Brewer, N., ZoAnetTi, J. y Young, R. L. (2017). The influence of media suggestions about links between criminality and autism spectrum disorder. Autism: The International Journal of Research and Practice, 21(1), 117-121. https://doi.org/10.1177/1362361316632097.

Briant, E., Watson, N. y Philo, G. (2013). Reporting disability in the age of austerity: the changing face of media representation of disability and disabled people in the United Kingdom and the creation of new 'folk devils'. Disability and Society, 28(6), 874-889. https:// doi.org/10.1080/09687599.2013.813837.

Christensen, D. L., Bilder, D. A., Zahorodny, W., Pettygrove, S., Durkin, M. S., Fitzgerald, R. T., ... y Yeargin-Allsopp, M. (2016). Prevalence and characteristics of autism spectrum disorder among 4-year-old children in the autism and developmental disabilities monitoring network. Journal of Developmental and Behavioral Pediatrics, 37(1), 1-8. https://doi.org/10.1097/DBP.0000000000000235.

Cuesta Suárez, H. N., Marchena Gómez, M. R. y Aguiar Perera, M. V. (2015). Percepciones sobre la discapacidad transmitidas por la prensa escrita española durante 1978, 1995 y 2007. Estudios sobre el Mensaje Periodistico, 21(1), 269-282. https://doi.org/10.5209/ rev_ESMP.2015.v21.n1.49094.

Doja, A. y RoberTs, W. (2006). Immunizations and autism: a review of the literature. Canadian Journal of Neurological Sciences, 33(4),341-346. https://doi.org/10.1017/S031716710000528X.

Dorries, B. y Haller, B. (2001). The news of inclusive education: a narrative analysis. Disability and Society, 16(6), 871-891. https://doi.org/10.1080/09687590120084001.

El País. Archivo web. Recuperado (02/12/2017) de https://elpais.com/archivo/.

Gómez, Y., Martín, M. y Castellet, A. (2014). Tratamiento de la discapacidad en el marco auto-normativo de los medios de comunicación españoles. Historia y Comunicación Social, 19, 667-679. https://doi.org/10.5209/rev_HICS.2014.v19.44993.

González, M. E., Montes, I. y Paniagua, F. J. (2018). Opiniones de los profesionales de la comunicación sobre el tratamiento de la discapacidad en televisión. Observatorio (OBS*), 12(1), 217-233. https://doi.org/10.15847/obsOBS12120181078.

Haller, B. A. (2010). Representing disability in an ableist world: essays on mass media. Louisville: Advocado Press.

Halliwell, M. (2017). Voices of mental health: medicine, politics, and American culture, 19702000. Rutgers University Press, Piscataway, NJ. https://doi.org/10.2307/j.ctt1vz498m.

Hanson, R. E. (2016). Mass communication: living in a media world. Londres: Sage Publications.

Hebert, E. B. y Koulouglioti, C. (2010). Parental beliefs about cause and course of their child's autism and outcomes of their beliefs: A review of the literature. Issues in comprebensive pediatric nursing, 33(3), 149-163. https://doi.org/10.3109/01460862.2010.498331.

JonEs, C. T. (2014). 'Why this story over a hundred others of the day?' five journalists' backstories about writing disability in Toronto. Disability and Society, 29(8), 1206-1220. https:// doi.org/10.1080/09687599.2014.916608.

Jones, S. C. y Harwood, V. (2009). Representations of autism in Australian print media. Disability and Society, 24(1), 5-18. https://doi.org/10.1080/09687590802535345.

Landis, J. R. y Koch, G. G. (1977). The measurement of observer agreement for categorical data. Biometrics, 33(1), 159-174. https://doi.org/10.2307/2529310.

Lanovaz, M. J., Dufour, M. y Shah, S. (2015). Newspaper coverage of autism treatment in Canada: 10-year trends (2004-2013). The Canadian Journal of Psychiatry, 60(7), 329-333. https://doi.org/10.1177/070674371506000706.

Ediciones Universidad de Salamanca / CC BY-NC-ND

Siglo Cero, vol. 51 (1), 2020, enero-marzo, pp. 73-87

$$
-86-
$$


McСombs, M. (2018). Setting the agenda: mass media and public opinion. Cambridge: Polity Press.

Morgan, J. (2018). Has autism found a place in mainstream TV? The Lancet Neurology, 17(10), 844. https://doi.org/10.1016/S1474-4422(18)30236-9.

Murray, S. (2007). Hollywood and the fascination of autism. En M. Osteen (Ed.), Autism and representation (pp. 244-255). N. York: Routledge.

Renwick, R. (2016). Rarely seen, seldom heard: people with intellectual disabilities in the mass media. En K. Scior y S. Werner (Eds.), Intellectual disability and stigma: Stepping out from the margins (pp. 61-75). Nueva York: Palgrave Macmillan. https://doi.org/10.1057/978-1137-52499-7_5.

Stemler, S. (2001). An overview of content analysis. Practical Assessment, Research and Evaluation, 7, 137-146.

Taylor, L. E., Swerdfeger, A. L. y Eslick, G. D. (2014). Vaccines are not associated with autism: an evidence-based meta-analysis of case-control and cohort studies. Vaccine, 32(29), 3623-3629. https://doi.org/10.1016/j.vaccine.2014.04.085.

URIEN, T. (2016). Del reconocimiento legal al reconocimiento efectivo de la igual dignidad como un derecho de la persona con discapacidad intelectual o del desarrollo. Un proceso que nos interpela. Siglo Cero, 47(2), 43-62. https://doi.org/10.14201/scero20164724362.

White, S. G., Meloy, J. R., Mohandie, K. y Kienlen, K. (2017). Autism spectrum disorder and violence: threat assessment issues. Journal of Threat Assessment and Management, 4(3), 144-163. https://doi.org/10.1037/tam0000089.

Wilson, K., Mills, E., Ross, C., McGowan, J. y Jadad, A. (2003). Association of autistic spectrum disorder and the measles, mumps, and rubella vaccine: a systematic review of current epidemiological evidence. Archives of Pediatrics and Adolescent Medicine, 157(7), 628-634. https://doi.org/10.1001/archpedi.157.7.628. 


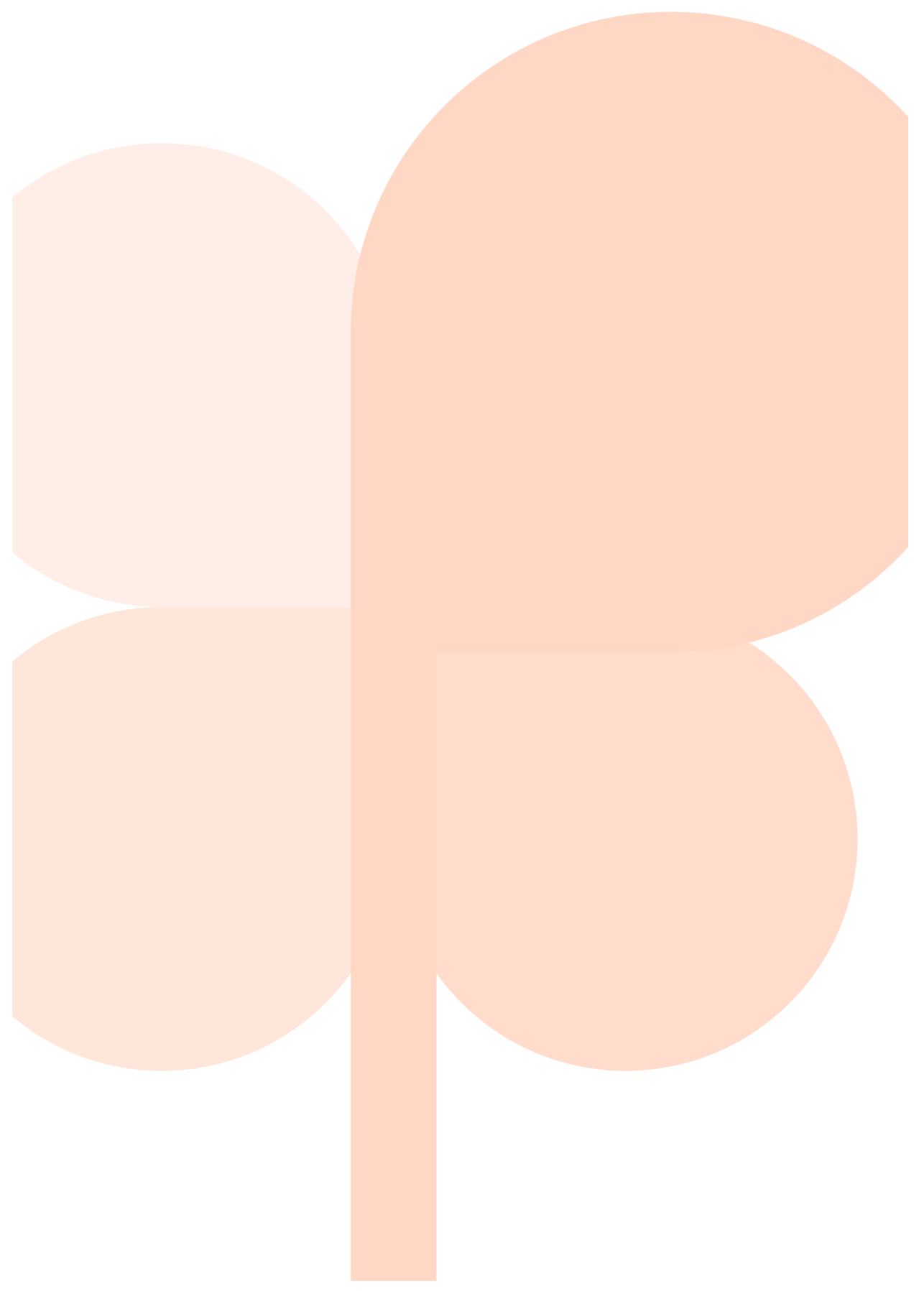

